Conclusion: In this validation study, the molecular signature of non-response identified patients who did not fulfill the EULAR good response criteria to TNFi therapies. The patient selection process for this study had limitations; additional analysis in an alternative cohort would further verify the performance of the MSRC test. Nevertheless, the test, previously validated for ACR50, now has been validated using EULAR good response in a treat-to-target setting.

REFERENCES:

[1] Schipper LG et al, Time to achieve remission determines time to be in remission. Arthritis Res Ther 201

[2] Mellors T, et al. Clinical Validation of a Blood-Based Predictive Test for Stratification of Response to Tumor Necrosis Factor Inhibitor Therapies in Rheumatoid Arthritis Patients. Network and Systems Medicine 2020

[3] Tweehuysen $L$ et al. Predictive value of ex-vivo drug-inhibited cytokine production for clinical response to biologic DMARD therapy in rheumatoid arthritis. Clin Exp Rheumatol 2019

Disclosure of Interests: Lixia Zhang Shareholder of: Scipher Medicine Corporation, Employee of: Scipher Medicine Corporation, Celeste van der Tog: None declared, Alfons den Broeder Consultant of: Abbvie, Amgen, Cellgene, Roche, Biogen, Lilly, Novartis, Celltrion Sanofi, Gilead., Grant/research support from: Abbvie, Amgen, Cellgene, Roche, Biogen, Lilly, Novartis, Celltrion Sanofi, Gilead., Ted Mellors Shareholder of: Scipher Medicine Corporation, Employee of: Scipher Medicine Corporation, Erin Connolly-Strong Shareholder of: Scipher Medicine Corporation, Employee of: Scipher Medicine Corporation, Johanna Withers Shareholder of: Scipher Medicine Corporation, Employee of: Scipher Medicine Corporation, Alex Jones Shareholder of: Scipher Medicine Corporation, Employee of: Scipher Medicine Corporation, Viatcheslav Akmaev Shareholder of: Scipher Medicine Corporation, Employee of: Scipher Medicine Corporation DOI: 10.1136/annrheumdis-2021-eular.3396

\section{POS0493 \\ TRIPLE-POSITIVITY AND ANTINUCLEAR ANTIBODIES, INSIGHTS ON DISEASE ACTIVITY: A RETROSPECTIVE COHORT STUDY OF COLOMBIAN RA PATIENTS}

K. Maldonado-Cañón ${ }^{1,2}$, D. V. Verano Castillo ${ }^{1}$, H. F. Arenas Torrado ${ }^{1}$, P. Coral Alvarado $^{2,3}$, P. A. Mendez Patarroyo ${ }^{2,3}$, G. Quintana Lopez ${ }^{2,3,4}$ on behalf of Reumavance Research Group. ${ }^{1}$ School of Medicine - National University of Colombia, Reumavance Research Group, Bogota, Colombia; ${ }^{2}$ Fundacion Santa Fe de Bogota University Hospital, Reumavance Research Group. Internal Medicine Department., Bogotá, Colombia; ${ }^{3}$ School of Medicine - Universidad de Los Andes, Reumavance research group. Internal Medicine Department, Bogota, Colombia; ${ }^{4}$ School of Medicine - National University of Colombia, Reumavance research group. Internal Medicine Department., Bogota, Colombia

Background: Rheumatoid arthritis (RA) is a chronic autoimmune inflammatory disease characterized by cartilage and bone destruction, which can lead to joint dysfunction and disability. Antibodies' role as biomarkers in RA has been recently increasing: Anti-citrullinated protein antibodies (ACPAs) are the most specific one $(60-70 \%$ of cases); whereas rheumatoid factor (RF) is seen in nearly $70 \%$ of cases, however, it is less specific than ACPA for RA diagnosis'. ACPA/RF positivity is related to a more severe phenotype and a rapid progression to clinically apparent RA $A^{\mathrm{ii}}$. Other biomarkers are Antinuclear Antibodies (ANAs), which have been related to a worse response to bDMARD treatment $\mathrm{tiii}^{\mathrm{ii}}$.

iNishimura K, Sugiyama D, Kogata Y, Tsuji G, Nakazawa T, Kawano S, et al. Meta-analysis: Diagnostic accuracy of anti-cyclic citrullinated peptide antibody and rheumatoid factor for rheumatoid arthritis. Vol. 146, Annals of Internal Medicine. American College of Physicians; 2007. p. 797-808

iiLingampalli N, Sokolove J, Lahey LJ, et al. Combination of anti-citrullinated protein antibodies and rheumatoid factor is associated with increased systemic inflammatory mediators and more rapid progression from preclinical to clinical rheumatoid arthritis. Clin Immunol. 2018;195:119-126.

iiilshikawa Y, Hashimoto M, Ito H, Tanaka M, Yukawa N, Fujii T, et al. Anti-nuclear antibody development is associated with poor treatment response to biological disease-modifying anti-rheumatic drugs in patients with rheumatoid arthritis. Semin Arthritis Rheum. 2019;49(2):204-10.

Objectives: Our aim was to compare in terms of mean differences the disease activity according to the presence of RF, ACPA, and ANA in an outpatient clinic-based cohort of Colombian RA patients.

Methods: We conducted a retrospective cohort study with clinical-epidemiological data obtained from May 2013 to Feb. 2020 of patients with RA diagnosis based on the 2010 ACR/EULAR classification criteria. The patients were stratified into eight subgroups according to their autoantibody status. Disease activity, assessed by the DAS28-ESR, was recorded at baseline and after $3, \approx 12, \approx$ 24 , and $\approx 36(+/-3)$ months. Mean DAS28-ESR differences were calculated by applying the Wilcoxon non-parametric rank test for two independent samples.

Results: Data of 384 patients who all completed 36 months of follow-up, from an ongoing cohort of $\approx 1100$ patients, were included in the analysis. On our primarily female $(n=294,76,8 \%)$ population, $\mathrm{RF}^{+} / \mathrm{ACPA}^{+} / \mathrm{ANA}^{+}$subgroup was the most prevalent $(n=183 ; 47,8 \%)$; interestingly, it was the one with the highest disease activity at baseline. After three months, all showed disease activity reduction; however, when completing follow-up, triple-positive, triple-negative and ANA-positive patients did not reach remission. Statistically significant mean differences were displayed when comparing overall and baseline mean DAS28ESR scores for $\mathrm{ANA}^{+}$vs ANA patients, as shown in table 1.

Table 1. Disease activity mean differences when comparing $\mathrm{ANA}^{+}$vs ANA $^{-}$patients

\begin{tabular}{|c|c|c|c|c|}
\hline & ANA $^{+}(n=218)$ & ANA $^{-}(n=165)$ & Mean difference & $p$ value \\
\hline Baseline & 4,05 & 3,62 & 0,43 & 0,014 \\
\hline 3 & 3,32 & 3,14 & 0,18 & 0,396 \\
\hline 12 & 3,13 & 2,89 & 0,24 & 0,059 \\
\hline 24 & 2,80 & 2,68 & 0,11 & 0,563 \\
\hline 36 & 2,80 & 2,58 & 0,22 & 0,098 \\
\hline Overall & 3,22 & 2,98 & 0,24 & 0,002 \\
\hline
\end{tabular}

Conclusion: In our study population, triple-positive and $\mathrm{ACPA}^{+} / \mathrm{ANA}^{+}$patients showed higher disease activity at baseline and on average during the follow-up period; furthermore, ANA positivity was shown to be conditional on a significant difference for higher disease activity. RF and ACPA positive have long since been described as determinants for disease activity; nonetheless, our research provides insights for the consideration of ANA titers as a novel addition that enables the preamble of triple-positivity as something to be acknowledged. Caution must be applied when interpreting these results, understanding the need for this matter to be subject of future research with greater sample size, and taking into account other potentially confounder variables.

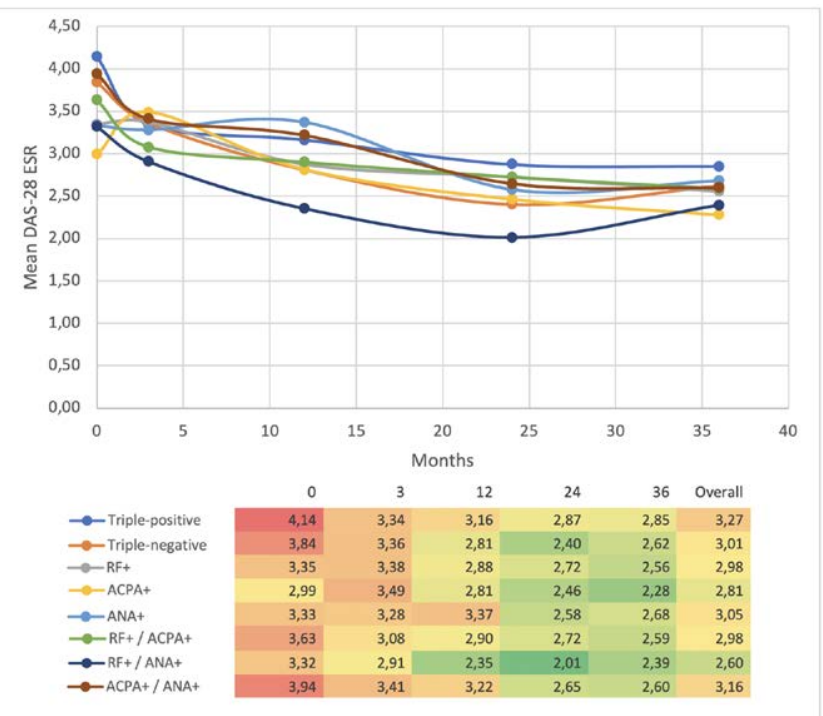

Figure 1. Mean disease activity for each stratum

Disclosure of Interests: None declared DOI: 10.1136/annrheumdis-2021-eular.3443

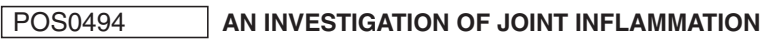 DEFINED BY COMPREHENSIVE ULTRASOUND ASSESSMENT AND PAIN SENSITISATION IN PATIENTS WITH ACTIVE RA}

K. Chaabo ${ }^{1}$, E. Chan ${ }^{2}$, T. Garrood ${ }^{2}$, Z. Rutter-Locher ${ }^{2}$, A. Vincent ${ }^{2}$, J. Galloway ${ }^{3,4}$, S. Norton ${ }^{5}$, B. Kirkham ${ }^{2}$. ${ }^{1}$ St George's Hospital, Rheumatology Department, London, United Kingdom; ${ }^{2}$ Guy's Hospital, Rheumatology Department, London, United Kingdom; ${ }^{3}$ King's College Hospital, Rheumatology Department, London, United Kingdom; ${ }^{4}$ King's College London, Rheumatology, London, United Kingdom; ${ }^{5}$ King's College London, Psychology and Inflammation Biology, London, United Kingdom

Background: Treat-to-target strategies have improved outcomes in patients with RA. Targets based on clinical assessments of tender (TJC) and swollen joints (SJC) were developed when most patients had active inflammatory disease. It is now recognised some patients have pain sensitisation often termed fibromyalgia (FM), making clinical assessment of inflammatory disease more complex. An indicator of pain sensitisation is the difference between TJC and SJC, an observation we described in a pilot study (1) 
Objectives: To study patients with RA to describe the relationships between enhanced clinical and patient-reported outcome measures (PROMS) and active inflammatory joint disease detected by comprehensive PDUS.

Methods: 158 sequential patients with RA with a DAS28ESR score $>\neg 3.2$ were enrolled from GSTT Rheumatology after informed consent. They were assessed by a rheumatologist for $66 \mathrm{SJC}$, 68TJC, fibromyalgia tender points \& physician global. Recorded PROMS were: patient global, widespread pain index (WPI), symptom severity score (somatic \& fatigue), FACIT fatigue, HAQ, EQ5D QoL, GAD7, PHQ9 and PHQ15. All patients had a 44 joint US scored for PD and grey scale by the EULAR scale blind to all patient data. Data were recorded on MedSciNet and statistical analysis used STATA.

Results: Patients meeting the ACR 2010 criteria for FM2(n=72) vs those that did not $(n=86)$ had similar demographics. SJC and US doppler scores were higher in the non-FM group, whereas rheumatoid factor, DAS28 and PROMs were higher in the FM group. Analysis of group differences using multinomial logistic regression and subsequent regularised (lasso) logistic regression, showed the FM group related to higher 28TJC, FACIT-F and PHQ15, with the non-FM group related to higher 28SJC, logESR, and lower Differences in 28S/TJC, Physician Global and FACIT-F. Patients were divided into four groups based on presence or absence of at least one PDUS+ joint and FM criteria; -FM-PD, -FM+PD, +FMPD and +FM+PD, data summarised Table 1. The -FM-PD and -FM+PD groups related to Physician Global and negatively related to FACIT-F, Difference in 28S/ TJC, and PHQ15 (-FM-PD). The +FM-PD group related positively to FACIT-F, Difference 28S/TJC, PHQ15, and negatively to Physician Global with +FM+PD related to PHQ9, FACIT-F and DAS28ESR

TABLE 1. -FM -PD -FM +PD +FM -PD +FM +PD p-value

\begin{tabular}{lccccc}
\hline Female & $34(79 \%)$ & $33(77 \%)$ & $35(83 \%)$ & $22(73 \%)$ & 0.77 \\
\hline $\begin{array}{l}\text { Total power } \\
\quad \text { doppler score }\end{array}$ & $0.0(0.0-1.0)$ & $16.0(10.0-25.0)$ & $0.0(0.0-0.0)$ & $9.0(8.0-20.0)$ & $<0.001$ \\
$\begin{array}{l}\text { Total gray } \\
\quad \text { scale score }\end{array}$ & $40.0(34.0-48.0)$ & $57.5(45.0-82.0)$ & $34.5(20.0-43.0)$ & $60.0(46.0-84.0)$ & $<0.001$ \\
Total Swollen JC & $3.0(1.0-5.0)$ & $6.0(3.0-9.0)$ & $2.0(0.0-5.0)$ & $5.5(2.0-10.5)$ & $<0.001$ \\
Total Tender & $5.5(3.0-12.0)$ & $8.0(4.0-11.0)$ & $13.0(8.0-20.0)$ & $15.0(8.0-20.0)$ & $<0.001$ \\
Tender-Swollen & $4.0(0.0-8.0)$ & $2.0(0.0-4.0)$ & $8.0(4.0-18.0)$ & $5.5(2.5-13.5)$ & $<0.001$ \\
$\quad$ JC Difference & & & & & \\
ESR mm/hour & $10.0(8.0-16.0)$ & $14.0(10.0-30.0)$ & $12.0(7.0-23.0)$ & $19.5(11.5-42.5)$ & 0.021 \\
Pain VAS & $50.0(40.0-65.0)$ & $50.0(30.0-60.0)$ & $60.0(47.5-77.5)$ & $67.5(55.0-80.0)$ & $<0.001$ \\
Patient VAS & $50.0(30.0-70.0)$ & $40.0(30.0-70.0)$ & $60.0(40.0-80.0)$ & $65.0(50.0-80.0)$ & 0.006 \\
Physician VAS & $30.0(20.0-50.0)$ & $50.0(40.0-60.0)$ & $30.0(20.0-40.0)$ & $50.0(32.5-70.0)$ & $<0.001$ \\
DAS28 (ESR) & $4.2(3.7-4.9)$ & $4.7(4.1-5.2)$ & $4.7(3.9-5.8)$ & $5.6(4.9-6.6)$ & $<0.001$ \\
& & & & &
\end{tabular}

Conclusion: These data using comprehensive US assessment to detect inflammatory joint disease, demonstrate that active inflammation defined as at least 1PDUS + joint, may account for disease activity in slightly less than half of unselected patients with RA with a DAS28ESR score $>3.2$. This study shows how frequently pain sensitisation accounts for symptoms and signs in RA. We suggest in addition to central sensitisation, often called FM, another group identified here as -FM-PD, may have pain from joint damage or peripheral pain sensitisation of joints, likely due to different mechanisms than central pain sensitisation. REFERENCES:

[1] Mian A et al, BMC Musculoskelet Disord. 2016;17: 404

[2] Wolfe $\mathrm{F}$ et al, Arth Care \& Res 2010; 62:600

Disclosure of Interests: Khaldoun Chaabo: None declared, Estee Chan: None declared, Toby Garrood: None declared, Zoe Rutter-Locher: None declared, Alexandra Vincent: None declared, James Galloway Speakers bureau: Dr Galloway has received speaker fees / honoraria from Abbvie, BMS, Celgene, Chugai, Gilead, Janssen, Lilly, Novartis, Pfizer, Roche, Sanofi, Sobi and UCB, Sam Norton: None declared, Bruce Kirkham Speakers bureau: Professor Bruce Kirkham has received speaker fees / honoraria from Abbvie, Gilead, Janssen, Lilly, Novartis, Pfizer,and UCB., Grant/research support from: Professor Bruce Kirkham has received research support from Lilly, Novartis and UCB.

Professor Bruce Kirkham has received speaker fees / honoraria from Abbvie, Gilead, Janssen, Lilly, Novartis, Pfizer and UCB.

DOI: 10.1136/annrheumdis-2021-eular.3509

\section{POS0495 LARGE JOINT DISEASE IN RHEUMATOID ARTHRITIS AND THE ROLE OF RHEUMATOID FACTOR. RESULTS FROM THE EARLY RHEUMATOID ARTHRITIS STUDY}

S. S. Zhao ${ }^{1}$, E. Nikiphorou ${ }^{2}$, A. Young ${ }^{3}$, P. Kiely ${ }^{4,5} .{ }^{1}$ University of Liverpool, Institute of Life Course and Medical Sciences, Liverpool, United Kingdom; ${ }^{2}$ King's College London, Centre for Rheumatic Diseases, London, United Kingdom; ${ }^{3}$ University of Hertfordshire, Center for Health Services and Clinical Research and Post Graduate Medicine, Hatfield, United Kingdom; ${ }^{4} S t$ George's, University of London, Institute of Medical and Biomedical Education,
London, United Kingdom; ${ }^{5}$ St George's University Hospitals NHS Foundation Trust, Rheumatology, London, United Kingdom

Background: Rheumatoid arthritis (RA) is classically described as a symmetric small joint polyarthritis with additional involvement of large joints. There is a paucity of information concerning the time course of damage in large joints, such as shoulder, elbow, hip, knee and ankle, from early to established RA, or of the influence of Rheumatoid Factor (RF) status. There is a historic perception that patients who do not have RF follow a milder less destructive course, which might promote less aggressive treatment strategies in RF-negative patients. The historic nature of the Ealy Rheumatoid Arthritis Study (ERAS) provides a unique opportunity to study RA in the context of less aggressive treatment strategies. Objectives: To examine the progression of large joint involvement from early to established RA in terms of range of movement (ROM) and time to joint surgery, according to the presence of RF.

Methods: ERAS was a multi-centre inception cohort of newly diagnosed RA patients (<2 years disease duration, csDMARD naive), recruited from 1985-2001 with yearly follow-up for up to 25 (median 10) years. First line treatment was csDMARD monotherapy with/without steroids, favouring sulphasalazine for the majority. Outcome data was recorded at baseline, at 12 months and then once yearly. Patients were deemed RF negative if all repeated assessments were negative. ROM of individual shoulder, elbow, wrist, hip, knee, ankle and hindfeet joints was collected at 3,5, 9 and 12-15 years. The rate of progression from normal to any loss of ROM, from years 3 to 14 was modelled using GEE, adjusting for confounders. Radiographs of wrists taken at years $0,1,2,3,5,7,9$ were scored according to the Larsen method. Change in the Larsen wrist damage score was modelled using GEE as a continuous variable, while the erosion score was dichotomised into present/absent. Surgical procedure data were obtained by linking to Hospital Episodes Statistics and the National Joint Registry. Time to joint surgery was analysed using multivariable Cox models.

Results: A total of 1458 patients from the ERAS cohort were included $(66 \%$ female, mean age 55 years) and $74 \%$ were RF-positive. The prevalence of any loss of ROM, from year 3 through to 14 was highest in the wrist followed by ankle, knee, elbow and hip. The proportion of patients at year 9 with greater than $25 \%$ loss of ROM was: wrist $30 \%$, ankle $12 \%$, elbow $7 \%$, knee $7 \%$ and hip $5 \%$. Odds of loss of ROM increased over time in all joint regions, at around 7 to $13 \%$ per year from year 3 to 14. There was no significant difference between RF-positive and RF-negative patients (see Figure 1). Larsen erosion and damage scores at the wrists progressed in all patients; annual odds of developing any erosions were higher in RF-positives OR $1.28(95 \% \mathrm{Cl} 1.24-1.32)$ than RF-negatives OR $1.17(95 \% \mathrm{Cl} 1.09-1.26)$, p 0.013 . Time to surgery was similar according to RF-status for the wrist and ankle, but RF-positive cases had a lower hazard of surgery at the elbow (HR 0.37, 0.15-0.90), hip (HR 0.69, 0.48-0.99) and after 10 years at the knee (HR 0.41, 0.25-0.68). Adjustment of the models for Lawrence assessed osteoarthritis of hand and feet radiographs did not influence these results.

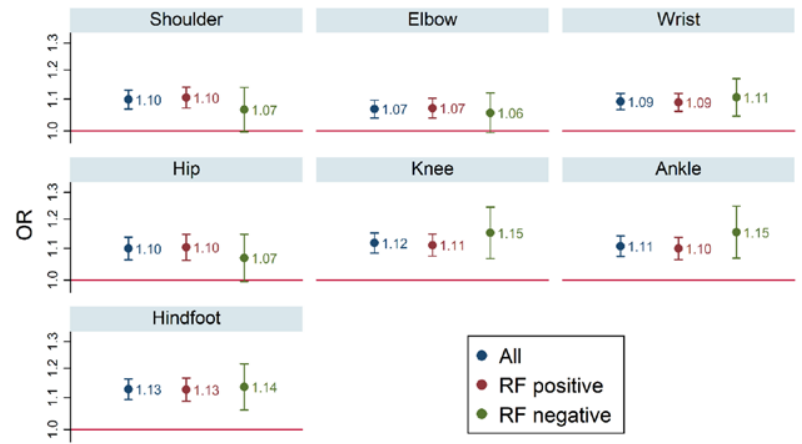

Figure 1. Odds of progression to any loss of ROM (from no loss of ROM) per year in the overall population and stratified by RF status.

Conclusion: Large joints become progressively involved in RA, most frequently affecting the wrist followed by ankle, which is overlooked in some composite disease activity indices. We confirm a higher burden of erosions and damage at the wrists in RF-positive patients, but have not found RF-negative patients to have a better prognosis over time with respect to involvement of other large joints. In contrast RF-negative patients had more joint surgery at the elbow, hip, and knee after 10 years. There is no justification to adopt a less aggressive treatment strategy for RF-negative RA. High vigilance and treat-to-target approaches should be followed irrespective of RF status.

Disclosure of Interests: None declared

DOI: 10.1136/annrheumdis-2021-eular.3626 